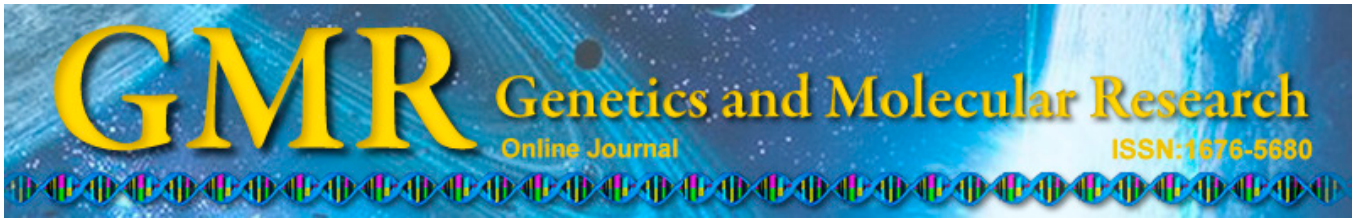

\title{
Characterization of a novel CAPN3 transcript generated by alternative splicing in cattle
}

\author{
S.Y. Liu ${ }^{1,2,3}$, H. Jiang ${ }^{1,2}$, B. Yuan ${ }^{1,2}$, Y. Gao ${ }^{1,2}$, L.S. Dai ${ }^{1,2}$ and J.B. Zhang ${ }^{1,2,3}$ \\ ${ }^{1}$ College of Animal Sciences, Jilin University, Changchun, Jilin, China \\ ${ }^{2}$ Laboratory Animal Center, Jilin University, Changchun, Jilin, China \\ ${ }^{3}$ Jilin Provincial Key Laboratory of Animal Embryo Engineering, Jilin University, \\ Changchun, Jilin, China \\ Corresponding authors: J.B. Zhang / L.S. Dai \\ E-mail: zjb515@163.com/1s_dai@163.com
}

Genet. Mol. Res. 14 (1): 457-463 (2015)

Received March 3, 2014

Accepted October 15, 2014

Published January 23, 2015

DOI http://dx.doi.org/10.4238/2015.January.23.20

\begin{abstract}
Calpain-3 (CAPN3) is a member of the calpain family of $\mathrm{Ca}^{2+}$-regulated cysteine proteases, which play an important role in sarcomere remodeling and mitochondrial protein turnover, and thus, regulating beef tenderness in cattle. Currently, multiple CAPN3 transcripts have been detected in human, monkey, rat, and rabbit. However, whether this transcript is present in cattle remains unknown. In this study, we identified 2 CAPN3 transcripts in the skeletal muscle individuals of local black cattle from Jilin, China. One transcript corresponded to the known full-length protein and was referred to as CAPN3a, while the second transcript did not contain exons 2-19 and contained a single-nucleotide insert in the penultimate base of exon 1 compared to CAPN3a; this protein was referred to as CAPN3b. The expression level of CAPN3b was approximately 50 -fold lower than that of CAPN3a. Moreover, CAPN3b mRNA was not translated into a functional protein because it had lost essential domains according to bioinformatic analysis. Our results not provide a foundation for understanding the function
\end{abstract}


of CAPN3, but also are useful for further elucidating the effect of CAPN3 on meat quality in cattle.

Key words: Alternative splicing; CAPN3; Cattle; Transcript

\section{INTRODUCTION}

Calpain-3 (CAPN3), also known as p94, is a member of the calpain family of $\mathrm{Ca}^{2+}$-regulated cysteine proteases. Calpains play important roles in many intracellular processes, including cell motility, cell multiplication, cell apoptosis, and muscle atrophy, by adjusting the biological activity of their substrates to control protein hydrolysis (Roperto et al., 2010; Paco et al., 2012; Felicio et al., 2013; Wang et al., 2013). The mammalian CAPN3 is most highly expressed in the skeletal muscle where its transcript abundance is approximately 10 times greater than that of other family members. CAPN3 shows a similar domain structure to the large-subunit of $\mu$-calpain and m-calpain (Goll et al., 2003). Furthermore, this domain contains 3 exclusive insertion sequences: a novel sequence at the N-terminus, an insertion sequence 1 (IS1) in domain II, and an insertion sequence 2 (IS2) between domains III and IV (Sorimachi et al., 1990).

Analysis of the mammalian CAPN3 gene (including human, rat, cattle, and monkey) revealed that it contains 24 exons. The IS1 region is coded by exon 6 , while the IS 2 region is coded by exons 15 and 16 (Nakajima et al., 2001). Currently, multiple transcripts produced by $C A P N 3$ have been detected in muscle and other tissues in human, monkey, rat, and rabbit (Azuma et al., 2000; Ma et al., 2000; Salem et al., 2011). In rodents, many types of splice variants of CAPN3 mRNA have been described; those characterized on the protein level include lens-specific Lp82 and Lp85 and retina-specific Rt88 (Shearer et al., 1998; Herasse et al., 1999; Ma et al., 2000). Lp82 contained a substitution of alternative exon 1 of a new N-terminus termed AX1, as well as deletions in exon 6, 15, and 16 (Ma et al., 1998);. Retina-specific Rt88 also contained the substituted AX1 sequence, but only exons 15 and 16 were deleted (Azuma et al., 2000). Moreover, many of these splice variants have been identified in humans. For instance, in the human lymphoblastoid cell line, exon 15 was spliced out organically, but only half of exon 6 had been spliced out (Herasse et al., 1999). Additionally, the deletion of exons 1-7 has been detected in humans as well as the novel insertion of Alu elements into the CAPN3 alter splicing sites within intron 7 (Salem et al., 2011). All the splice isoforms generally caused specific changes in CAPN3 function.

As in other species, CAPN3 plays an important role in sarcomere remodeling and mitochondrial protein turnover in cattle (Cohen et al., 2006; Robinson et al., 2012), thus regulating beef tenderness (Barendse et al., 2008). However, the CAPN3 mRNA splicing isoform in cattle remains unknown. Here, we report a new short CAPN3 transcript lost between exons 2 and 19 in an individual of the local black cattle of China. The current data provide a foundation for future studies examining how CAPN3 performs its essential function in the skeletal muscle of cattle.

\section{MATERIAL AND METHODS}

\section{Animals}

Six cattle breeds (Simmental, Charolais, Limousin, Angus, local yellow cattle, and local black cattle from Jilin of China) were used as the experimental population. The skeletal 
muscles (6 samples from each breed) were dissected in the local slaughter house, immediately frozen in liquid nitrogen, and then stored at $-80^{\circ} \mathrm{C}$ until analysis. Muscles from each animal were individually subjected to reverse transcription-polymerase chain reaction (PCR) to analyze the CAPN3 mRNA splicing isoform.

\section{RNA isolation}

Total RNA was extracted from frozen skeletal muscles of all cattle using TRIzol Reagent (Invitrogen, Carlsbad, CA, USA) according to manufacturer instructions. These RNA samples were dissolved in $0.1 \%$ diethylpyrocarbonate-treated water and the quality was evaluated by agarose gel electrophoresis and ethidium bromide staining. RNA samples were treated with the RNase-free DNase-I (Takara, Shiga, Japan) according to manufacturer instructions. RNA concentrations were determined using a Nanodrop ${ }^{\circledR}$ ND-1000 spectrophotometer (Thermo Fisher Scientific, Waltham, MA, USA). Total RNA was stored at $-80^{\circ} \mathrm{C}$ until use.

\section{RT-PCR amplification and quantification}

First-strand cDNA was reverse-transcribed using $1 \mu \mathrm{g}$ total RNA with AMV reverse transcriptase (Takara) and oligo-dT(18) primers in a $10-\mu \mathrm{L}$ volume. The reaction was carried out at $45^{\circ} \mathrm{C}$ for $45 \mathrm{~min}$. AMV reverse transcriptase was inactivated by incubation for $5 \mathrm{~min}$ at $95^{\circ} \mathrm{C}$. The cDNA products were stored at $-20^{\circ} \mathrm{C}$. PCR was carried out in a $25-\mu \mathrm{L}$ reaction volume using the cDNA as a template and Ex Taq polymerase (Takara). Using a pair of primers designed to correspond to the 5'-untranslated region (UTR) and 3'-UTR of the CAPN3 gene (Bos taurus CAPN3, GenBank accession No. NM_174260), we amplified nearly the entire CAPN3 mRNA (F: 5'-GCTGAATCTTGCTGTCTTTA-3'; R: 5'-CTTTGAGGTAAGTGAC TAGC-3'). The following PCR program was used: initial denaturation at $95^{\circ} \mathrm{C}$ for $45 \mathrm{~s}, 40$ cycles at $94^{\circ} \mathrm{C}$ for $35 \mathrm{~s}, 62^{\circ} \mathrm{C}$ for $75 \mathrm{~s}, 72^{\circ} \mathrm{C}$ for $3 \mathrm{~min}$, and a final extension at $72^{\circ} \mathrm{C}$ for $10 \mathrm{~min}$. PCR products were analyzed by $1.5 \%$ agarose gel electrophoresis and visualized by ethidium bromide staining. The intensities of substrate and cleavage product bands were quantified using the TotalLab Quant image analysis software (www.totallab.com/products/totallabquant/).

\section{Cloning and sequencing}

PCR products were purified using the columns included in the AxyPrep ${ }^{\mathrm{TM}}$ PCR Cleanup Kit (Axygen, Union City, CA, USA) and ligated into the pMD-18T vector (Takara) according to manufacturer recommendations. Recombinant plasmids were transformed into Escherichia coli competent cells (strain DH5 $\alpha$ ). At least 20 positive clones of each PCR product were sequenced. Sequencing was conducted on both strands using the pMD-18T vector primers at the Shanghai Sangon Biological Engineering Technology and Services Co., Ltd., China. Nucleotide sequence alignments, translations, and comparisons were analyzed using DNAMAN (Lynnon Corporation, Quebec, Canada) and the BLAST program at NCBI (http://www.ncbi.nlm.nih.gov/BLAST/).

\section{Statistical analysis}

Data are reported as means \pm standard error. The significance of the differences be- 
tween groups was determined by one-way analysis of variance with SPSS 16.0 (SPSS, Inc., Chicago, IL, USA).

\section{RESULTS AND DISCUSSION}

Total RNA isolated from skeletal muscles was analyzed by RT-PCR using full-length primers located at 5'- and 3'-UTR, which amplified nearly the entire CAPN3 mRNA. These results suggested that in addition to the expected fragment size of $2598 \mathrm{bp}$, which was detected in all of the cattle breeds examined, a low molecular weight fragment was also detected in some individuals of local black cattle from Jilin of China (Figure 1A). The longer transcript observed in all samples tested was referred to as CAPN3a, and the novel transcript identified in this study was referred to as CAPN3b. These results show that the genetic evolution of CAPN3 in the local black cattle from Jilin of China is different from that in other breeds.

The levels of each CAPN3 transcript were quantified from the same individual using the TotalLab Quant image analysis software (www.totallab.com/products/totallabquant/). The expression level of CAPN3b was approximately 50-fold lower than CAPN3b (Figure 1B). In human, mouse, rat, and rabbit, the levels of multiple alternative transcripts were also 10- to 1000-fold lower than the main product (Herasse et al., 1999; Kawabata et al., 2003).

A

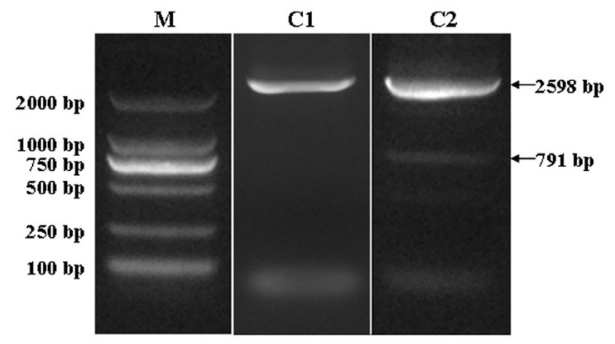

B

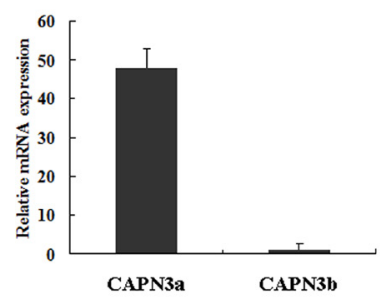

$\mathrm{C}$

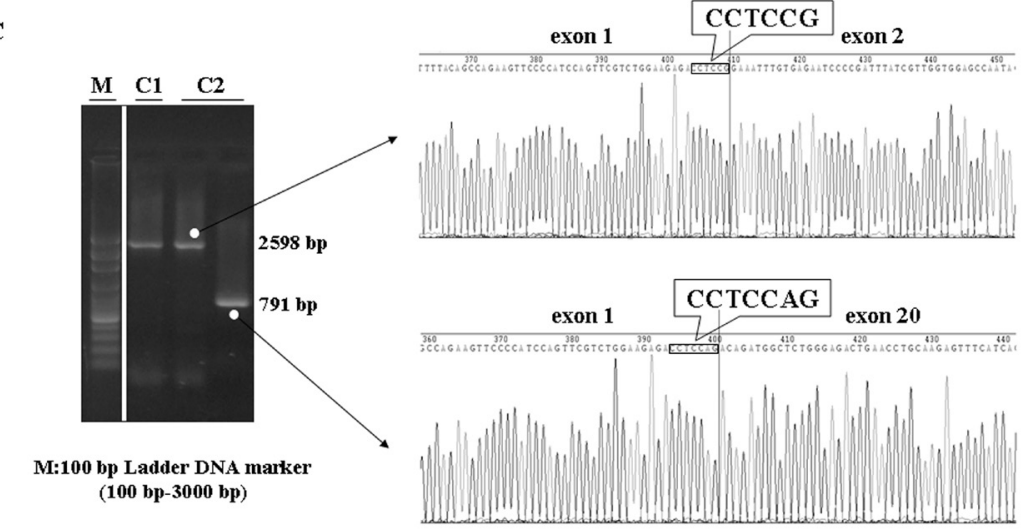

Figure 1. Cloning and sequence analysis of the novel CAPN3 transcript in cattle. A. Identification of a novel CAPN3 trasncript in cattle. The sizes of PCR fragments are indicated. The representive RT-PCR results of CAPN3 cDNA are shown. B. Relative mRNA quantification of CAPN3a and CAPN3b from the same individual. Data are reported as means \pm SE. C. Nucleotide sequence analysis of two different CAPN3 transcripts. The fragment of 2598 $\mathrm{bp}$ corresponds to the normal CAPN3 mRNA sequence. The fragment of $791 \mathrm{bp}$ is a novel identified transcript with deletion from exon 2 to exon 19 as well as a single-nucleotide insert in the penultimate of exon 1 . The variation of exon 1 between the two different transcripts is magnified in the sequencing data. The sequence of CCTCCAG in CAPN3b is identical with the sequence at the end of intron 19 of the CAPN3 gene. 
A schematic diagram of the 2 transcript variants of CAPN3 is shown in Figure 2A.

A

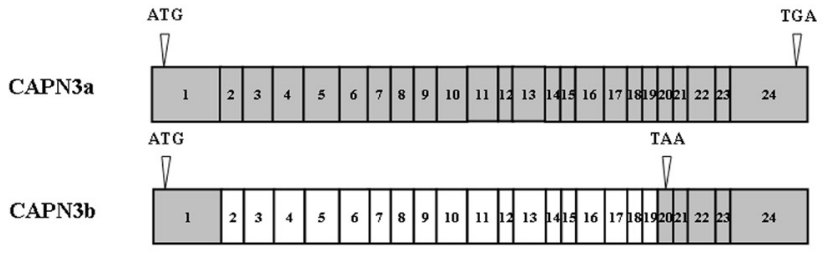

B

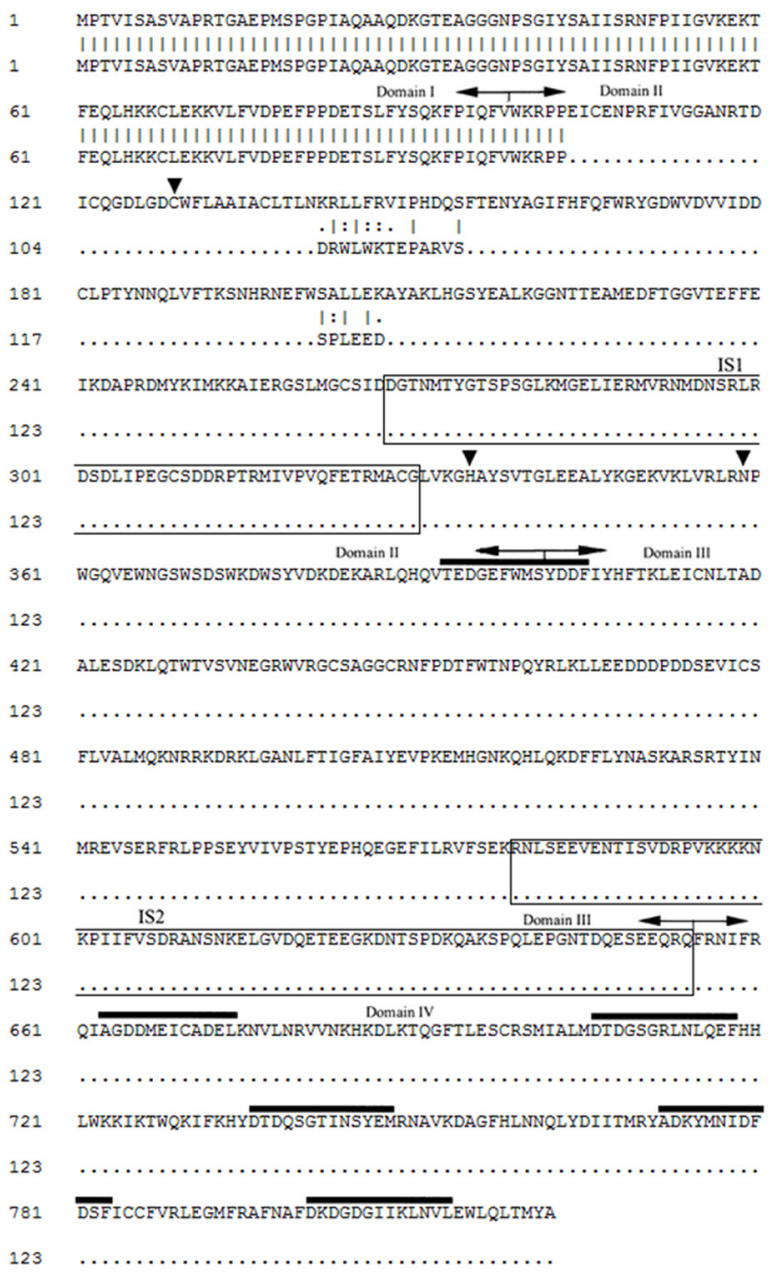

Figure 2. A. Schematic diagram of the two different CAPN3 transcripts. The number in the box represents the different exon. The start codon ATG and stop codon TGA/TAA are marked. The deletions of exons in the CAPN3b are indicated as white boxes. B. Amino acid sequence analysis of two different CAPN3 transcripts. The full-length amino acid sequence of CAPN3 was obtained from NM_174260. Arrows point out borders of domain structures. The amino acid sequences of IS1 and IS2 regions are indicated by the two boxes, respectively. The black thick lines indicate EF hand structures. The deletion sequence of CAPN3b is indicated as dashed line. 
Translation of the CAPN3a produced a 94-kDa protein of 822 amino acids. The protein contained a short $\mathrm{N}$-terminal region (domain I), a papain-type proteolytic domain (domain II), a C2-like domain (domain III), and a calcium-binding domain composed of EF hands (domain IV) (Nakajima et al., 2001; Duguez et al., 2006). However, the predicted CAPN3b amino acid sequence only contained the N-terminal 103 amino acids of the CAPN3a protein and an additional 19 amino acids because of the frame shift in the coding region, which lacked domains II, III, and IV (Figure 2B).

It has been reported that CAPN3 could not be degraded by autolysis immediately when the IS1 structure is deleted (Sorimachi et al., 1993; Herasse et al., 1999; Taveau et al., 2003). IS2 is encoded by exons 15 and 16. In COS-7 cells, the CAPN3 protein did not show nuclear localization when a CAPN3 splice variant lacking the basic PVKKKKNKP sequence was encoded by exon 15, suggesting that exon 15 plays a role as a nuclear translocation signal (Sorimachi et al., 1993). In addition, IS2 has been shown to be important for controlling CAPN3 activity, as exon 15 deletion leads to autolytic activity disorders, and exon 16 deletion leads to substrate proteolysis (Herasse et al., 1999). Therefore, CAPN3b mRNA is not translated into a functional protein because of the loss of essential domains, and may be transcribed as a long non-coding RNA. However, this requires further investigation.

Overall, we identified a novel CAPN3 transcript isoform generated by alternative splicing in cattle. This shorter variant showed low abundance in muscle tissues compared to the full-length protein. Moreover, the novel CAPN3 transcript identified appears to be a long non-coding RNA, as it cannot be translated into a functional protein. The current results not only provide a foundation for understanding the expression of CAPN3, but also provide useful information for further elucidating the effect of CAPN3 on meat quality in cattle.

\section{ACKNOWLEDGMENTS}

Research supported by the Earmarked Fund for Modern Agro-Industry Technology Research System (\#CARS-38) and the Innovative Research Team in University (Grant \#IRT1248) in China.

\section{REFERENCES}

Azuma M, Fukiage C, Higashine M, Nakajima T, et al. (2000). Identification and characterization of a retina-specific calpain (Rt88) from rat. Curr. Eye Res. 21: 710-720.

Barendse W, Harrison BE, Bunch RJ and Thomas MB (2008). Variation at the Calpain 3 gene is associated with meat tenderness in Zebu and composite breeds of cattle. BMC Genet. 9: 41.

Cohen N, Kudryashova E, Kramerova I, Anderson LV, et al. (2006). Identification of putative in vivo substrates of calpain 3 by comparative proteomics of overexpressing transgenic and nontransgenic mice. Proteomics 6: 6075-6084.

Duguez S, Bartoli M and Richard I (2006). Calpain 3: a key regulator of the sarcomere? FEBS J. 273: 3427-3436.

Felicio AM, Boschiero C, Balieiro JC, Ledur MC, et al. (2013). Identification and association of polymorphisms in CAPN1 and CAPN3 candidate genes related to performance and meat quality traits in chickens. Genet. Mol. Res. 12: $472-482$.

Goll DE, Thompson VF, Li H, Wei W, et al. (2003). The calpain system. Physiol. Rev. 83: 731-801.

Herasse M, Ono Y, Fougerousse F, Kimura E, et al. (1999). Expression and functional characteristics of calpain 3 isoforms generated through tissue-specific transcriptional and posttranscriptional events. Mol. Cell. Biol. 19: 4047-4055.

Kawabata Y, Hata S, Ono Y, Ito Y, et al. (2003). Newly identified exons encoding novel variants of p94/calpain 3 are expressed ubiquitously and overlap the $\alpha$-glucosidase C gene. FEBS Lett. 555: 623-630.

Ma H, Fukiage C, Azuma M and Shearer TR (1998). Cloning and expression of mRNA for calpain Lp82 from rat lens: splice variant of p94. Invest. Ophth. Vis. Sci. 39: 454-461. 
Ma H, Shih M, Hata I, Fukiage C, et al. (2000). Lp85 calpain is an enzymatically active rodent-specific isozyme of lens Lp82. Curr. Eye Res. 20: 183-189.

Nakajima T, Fukiage C, Azuma M, Ma H, et al. (2001). Different expression patterns for ubiquitous calpains and Capn3 splice variants in monkey ocular tissues. Biochim. Biophys. Acta 1519: 55-64.

Paco S, Ferrer I, Jou C, Cusí V, et al. (2012). Muscle fiber atrophy and regeneration coexist in collagen VI-deficient human muscle: role of calpain-3 and nuclear factor-кB signaling. J. Neuropath. Exp. Neur. 71: 894-906.

Robinson D, Cafe L, McIntyre B, Geesink G, et al. (2012). Production and processing studies on calpain-system gene markers for beef tenderness: consumer assessments of eating quality. J. Anim. Sci. 90: 2850-2860.

Roperto S, De Tullio R, Raso C, Stifanese R, et al. (2010). Calpain3 is expressed in a proteolitically active form in papillomavirus-associated urothelial tumors of the urinary bladder in cattle. PloS One 5: e10299.

Salem IH, Hsairi I, Mezghani N, Kenoun H, et al. (2011). CAPN3 mRNA processing alteration caused by splicing mutation associated with novel genomic rearrangement of Alu elements. J. Hum. Genet. 57: 92-100.

Shearer T, Ma H, Shih M, Hata I, et al. (1998). Lp82 calpain during rat lens maturation and cataract formation. Curr. Eye Res. 17: 1037-1043.

Sorimachi H, Ohmi S, Emori Y, Kawasaki H, et al. (1990). A novel member of the calcium-dependent cysteine protease family. Biol. Chem. Hoppe Sevler 371 (Suppl): 171-176.

Sorimachi H, Toyama-Sorimachi N, Saido T, Kawasaki H, et al. (1993). Muscle-specific calpain, p94, is degraded by autolysis immediately after translation, resulting in disappearance from muscle. J. Biol. Chem. 268: 10593-10605.

Taveau M, Bourg N, Sillon G, Roudaut C, et al. (2003). Calpain 3 is activated through autolysis within the active site and lyses sarcomeric and sarcolemmal components. Mol. Cell. Biol. 23: 9127-9135.

Wang CH, Liang WC, Minami N, Nishino I, et al. (2013). Limb-girdle muscular dystrophy type 2A with mutation in CAPN3: the first report in Taiwan. Pediatr. Neonatol. Doi: 10.1016/j.pedneo.2013.01.018. [Epub ahead of print]. 